Bangladesh Journal of Neuroscience 2012; Vol. 28 (1) : 45-51

\title{
The Sitting Position in Neurosurgery: A Clinical Study in 30 Cases
}

\author{
SHAMSUL ALAM ${ }^{1}$, ATM MOSSARAF HOSSAIN $^{2}$, REZAUL AMIN $^{3}$, ANM WAKIL $^{3}$, \\ KM TARIKUL ISLAM ${ }^{3}$, RUKUN UDDIN CHOWDHURY ${ }^{4}$.
}

\begin{abstract}
:
Objective: Sitting position for operation of posterior fossa lesions, occipital and posterior parietal lesions, foramen magnum, upper cervical spinal lesions provides an excellent visualization because of slack of brain due to gravity drainage of CSF and blood. Hence gross total tumour removal relatively easy and less complicative. Methods: From January 2008 to march 2010 total 30 cases underwent neurosurgical procedure in sitting position. Physical characteristics including patients age, sex, size of the tumour and histological diagnosis were collected. The post operative image were studied to see the extent of tumour removal and early detection of complications. Almost all patients required peroperative cerebral venous line or peripheral inserted central venous line, precordial doppler sound, $\mathrm{ETCO}_{2} \mathrm{O}_{2}$ saturation and close monitoring of blood pressure. Results: Venous air embolism were detected in two cases (6.6\%). Total tumour removal was possible in 17 (56.6\%) cases and subtotal in 11 (36.6\%) cases. There were 4 (13.33\%) mortality in thirty cases, two cases from CP angle tumour and another case from petroclival meningioma and another from pineal region tumour. There was pneumocephalus in almost all cases and post-operative new facial paresis in 10 (33.3\%) cases. Fifth cranial nerve palsy developed in 3 (10\%) cases. Temporary lower cranial nerve palsy developed in 2 cases. Post-operative tumour bed haematoma developed in 4 (13.33\%) cases. Most of the patient had good outcome (GOS 5). Conclusion: Sitting position can be safely done with good preoperative physiological, peroperative close monitoring of the patient regarding blood pressure, ETCO and oxygen saturation. However postoperative complication like tumour bed haematoma, pneumocephalus, cranial nerve palsy have to be bring in mind.
\end{abstract}

\section{Introduction:}

The use of the sitting or semisitting position for patients undergoing posterior fossa and/or cervical spine surgery facilitates easy surgical access but presents a physiological challenges for the anaesthetist ${ }^{1}$. This patient position provides optimum access to midline, paramedian and cerebellopontine lesions it also improves cerebral venous drainage, lowers intracranial pressure (ICP) and promotes gravity drainage of blood and cerebral spinal fluid (CSF) ${ }^{2,3}$.Complications related to the use of this position includes haemodynamic instability, venous air embolism (VAE), embolism related hypoxia and/or hypotension, with the possibility of paradoxical air embolism, pneumocephalus, quadriplegia and compressive peripheral neuropathy ${ }^{1-3}$. Alternative positions for surgical access to the posterior fossa and the cervical spine include the prone, park bench, lateral positions. Prolonged neurosurgical procedures with pin fixation of the head in semisitting positions necessitate extensive patient monitoring to ensure cardiorespiratory homeostasis ${ }^{1-3}$.

The objective of this study is to provide a riskbenefit analysis of the present day use of the sitting position for patients undergoing $\mathrm{CP}$ angle, midline posterior fossa and cervical spine surgery.

1. Assistant Professor, Department of Neurosurgery, Bangabandhu Sheikh Mujib Medical University, Dhaka.

2. Professor, Department of Neurosurgery, Bangabandhu Sheikh Mujib Medical University, Dhaka.

3. Resident, Department of Neurosurgery, Bangabandhu Sheikh Mujib Medical University, Dhaka.

4. Consultant, Department of Neurosurgery, Combined Military Hospital, Dhaka. 
Since 1913 when the first surgery with the patient in sitting position was performed, the debate concerning this positioning has continued. The sitting position is thought to be best for surgical access to $\mathrm{CP}$ angle, midline and paramedian posterior fossa or to posteriorly located parietal lesions ${ }^{4}$. Gravity facilitates drainage of blood and other fluids and an optimal view over the pathology is possible with lowered intracranial pressure and increased venous return ${ }^{1-4}$.

Air embolism can occur during any surgical procedure in which the operative site is $5 \mathrm{~cm}$ or higher above the right atrium ${ }^{4}$.

The higher chance of venous air embolism occur in the presence of persistent foramen ovale (PFO) ${ }^{5}$. In patient with $\mathrm{PFO}$ surgical opening of veins can lead to a paradoxical air embolism with critical cerebral and cardio-pulmonary complication. PFO have found in $28 \%$ of adult patients using transesophageal echocardiography as diagnostic procedure $^{5}$. Doppler ultrasound is the most sensitive noninvasive monitor, and is commonly used in sitting position. The monitor uses ultrahigh frequency sound waves (usually between 2 and 3 megahertz) to measure blood flow velocity and changes in blood density. This information is converted to a characteristic sound ${ }^{5,6}$.

Transesophageal echocardiography is more sensitive than Doppler ultrasound, and is also more invasive and technically more difficult to place and to interpret. It does, however, allow determination of the volume of air aspirated. Transesophageal echocardiography will also show air passing through a patent foramen ovale into the left atrium and into the systemic circulation ${ }^{5-7}$.

Monitoring of End-tidal carbon dioxide is commonly used, widely available, and sensitive. It is highly sensitive but not specific for air embolism. It becomes low at the beginning of venous air embolism. However hyperventilation, low cardiac output, other types of emboli, and COPD can also decrease $\mathrm{ETCO}^{7,8}$.

The least sensitive monitor is the precordial or esophageal stethoscope. A "millwheel murmur" indicates a massive air embolism. When a millwheel murmur is heard, cardiovascular collapse is imminent $^{7,8}$.
A multiorifice central venous catheter should be placed in patients at risk of air embolism. The optimal site for the tip of the catheter is at the SVCRA junction. If an embolus occurs, air can be aspirated through the catheter before it enters the pulmonary circulation ${ }^{5-8}$.

Treatment of air embolism is largely supportive. The surgeon should be informed as soon as the diagnosis is made. $\mathrm{N} 2 \mathrm{O}$ diffuses into air bubbles faster than nitrogen can diffuse out, and increases the size of the bubble. If $\mathrm{N} 2 \mathrm{O}$ is used, it should be discontinued when an air embolism occurs. FiO2 (fraction of inspired $\mathrm{O}_{2}$ ) should be increased to 1.0. The surgeon should flood the surgical field with fluids while open veins are cauterized or exposed bony emissary vein is waxed. If significant amounts of air have entered the circulation, the jugular veins should be manually occluded. This will prevent additional air from being entrained while the surgeons obtain hemostasis. The blood pressure should be supported with fluid and vasopressors ${ }^{8}$.

Controversy surrounds the use of the sitting position for neurosurgery, regarding the risk of venous air embolus (VAE) and its sequelae. The reported incidence of VAE in adults undergoing neurosurgery in the sitting position varies from 7 to $50 \%{ }^{8}$.

\section{Methods:}

From January 2008 to march 2010 total 30 cases underwent neurosurgical procedure in sitting position. Physical characteristics including patient's age, weight, and histological diagnosis were collected. The post-operative notes were studied together with the follow up notes to document any unexpected neurological sequelae. Almost all patients required $\mathrm{CV}$ line or peripheral inserted central venous catheter, precordial doppler sound, $\mathrm{ETCO}_{2}, \mathrm{O}_{2}$ saturation and close monitoring of blood pressure. Routine use of normal saline irrigation to the operation field, regular use of bone wax to the bone emissary vein, bilateral jugular vein compression before starting of dural incision to locate the transverse and sigmoid sinus and minimum cautery to the tumour bed. To achieve 
hemostasis we raise the blood pressure $20 \mathrm{~mm}$ of $\mathrm{Hg}$ above the preinduction blood pressure and again bilateral jugular vein compression to exclude any venous bleeding before closure of dura.

Internal acoustic meatus was Drilling of most of the cases of acoustic schwannomas. We didnot use facial nerve monitor to trace out the location of facial nerve because of its nonavailability in our institute. We did routine practice of raising the blood pressure before closing the dura and bilateral jugular vein compression to check the operative site bleeding. Drilled Internal acoustic meatus was filled by subcutaneous fat. Fibrin glue were used in some cases to make the the dural closure water tight. In some cases we used acrylic bone cement to close the lateral suboccipital craniectomy defect followed by suboccipital muscle and subcutaneous tissue and skin closure.

\section{Results:}

Demographic study from table I revealed age group between 25 and 65 (median 40, average 45), among them 16 (53.3\%) were male and 14 (46.7\%) were female patients (Table-II).

Table-I

Shows distribution of age group:

\begin{tabular}{lc}
\hline Age & No. of Patient \\
\hline $21-30$ & $3(10 \%)$ \\
$31-40$ & $10(33.3 \%)$ \\
$41-50$ & $9(30 \%)$ \\
$51-60$ & $7(23.3 \%)$ \\
$61-70$ & $1(3.3 \%)$ \\
\hline
\end{tabular}

Table-II

Shows distribution of sex group:

\begin{tabular}{lc}
\hline Sex & No. of Patient \\
\hline Male & $16(53.3 \%)$ \\
Female & $14(46.6 \%)$ \\
\hline
\end{tabular}

Headache and vomiting was presented in most of the cases (27 patients), deafness in 18 cases, facial weakness were in 3 patients, ataxia in 24 cases, quardriparesis were seen in 3 cases (Table-III).
Table-III

Shows distribution of presentation group:

\begin{tabular}{lc}
\hline Presentation & No. of Patients \\
\hline Headache & $27(90 \%)$ \\
Vomiting & $27(90 \%)$ \\
Deafness & $18(60 \%)$ \\
Facial Weakness & $5(16.6 \%)$ \\
Ataxia & $24(80 \%)$ \\
Quadriparesis & $3(10 \%)$ \\
\hline
\end{tabular}

$\mathrm{CP}$ angle tumour were $60 \%$, petroclival meningioma were $20 \%$, foramen lesions were $6.6 \%$, pineal region tumour were $10 \%$, upper cervical schawnnoma $3.3 \%$. Total tumour removal was possible in $11(36.6 \%)$ cases and subtotal in 17 (56.6\%) cases (table-IV).

Table-IV

Location of Lesions:

\begin{tabular}{lc}
\hline Location & No of Patients \\
\hline Cerebellopontine angle & $18(60 \%)$ \\
Petroclival Meningioma & $6(20 \%)$ \\
Foramen Magnum Schwannoma & $1(3.3 \%)$ \\
Foramen Magnum Meningioma & $1(3.3 \%)$ \\
Pineal Region Tumour & $3(10 \%)$ \\
Upper cervical Schawnnoma & $1(3.3 \%)$ \\
\hline Total & 30 \\
\hline
\end{tabular}

Gross total tumour removal was possible in 11 $(36.6 \%)$ cases(Figure 1,2,4,5) and subtotal removal in $17(56.6 \%)$ cases (figure $5,6,7,8)$ and partial removal in $2(6.66 \%)$ cases.

Facial nerve preservation could possible in 10 cases $(55 \%)$ (Figure 5,8$)$ and not possible in 8 cases (45\%) out of cases of $18 \mathrm{CP}$ angle tumour.

There were $4(13.33 \%)$ mortality in thirty cases, two cases from CP angle tumour one case from petroclival meningioma and another from pineal region tumour. There were pneumocephalus in almost all cases and post-operative new facial paresis in $13(13.3 \%)$ cases. $5^{\text {th }}$ nerve palsy developed in $3(10 \%)$ cases, lower cranial nerve palsy developed in 2 cases (6.6\%). Post-operative tumour bed haematoma developed in 4 (13.33\%) cases. Among them 2 cases underwent urgent reexploration and hematoma evacuation. Most of the patients have good outcome (GOS 5) (Table-V). 
Table-V

Complications of sitting position:

\begin{tabular}{lc}
\hline Complications & No. of Patients \\
\hline Facial nerve palsy & $10(33.3 \%)$ \\
$5^{\text {th }}$ nerve palsy & $3(10 \%)$ \\
Lower cranial nerve palsy & $2(6.6 \%)$ \\
Hematoma & $5(16.6 \%)$ \\
Hydrocephalus & $3(10 \%)$ \\
Cerebellar swelling & $3(10 \%)$ \\
Venous air embolism & $2(6.6 \%)$ \\
Pseudomeningocele & $3(10 \%)$ \\
Cervical cord injury & $\mathrm{Nil}$ \\
\hline
\end{tabular}

\section{Discussion:}

For many neurosurgeons the sitting position (Figure $1 \& 2$ ) offers the advantages of optimal surgical exposure, better anatomic orientation, improved cerebral venous drainage, lower intracranial pressure, and enhanced gravity drainage of blood and cerebrospinal fluid ${ }^{7}$. Because life threatening complications can occur during neurosurgery in the sitting position hence caution is advocated in almost all patients including patients with a patent foramen ovale, atherosclerotic cardiovascular disease, severe hypertension or cervical stenosis ${ }^{7,8}$.

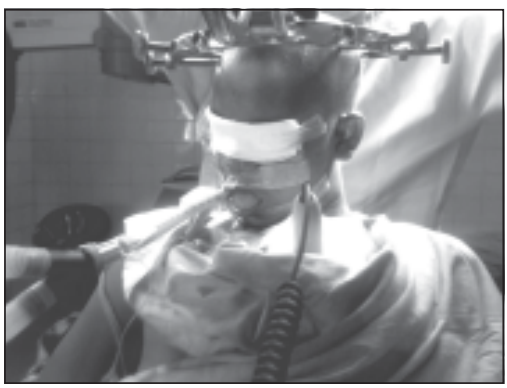

Fig.-1: Front view of sitting position with head fixation by 4 pin head fixator and precordial Doppler.

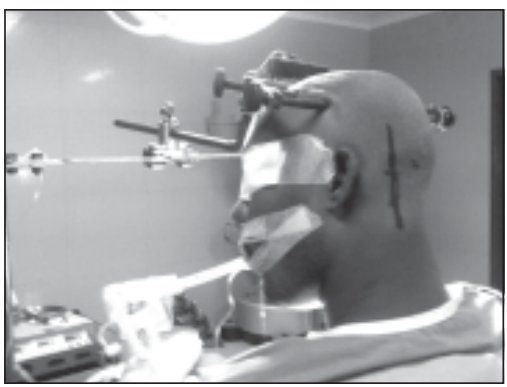

Fig.-2: Back view of sitting position.
Cervical cord injury is one of the most serious complications after surgery in sitting position. In 1980, Hitselberger and House reported five cases of midcervical quadriplegia after acoustic tumour resection performed with the patient in the sitting position. They suggested that acute diffuse infraction was caused by direct prolonged ${ }^{9}$. Pressure on cervical cord related to a preexisting spondylotic bar at the midcervical level ${ }^{9}$.

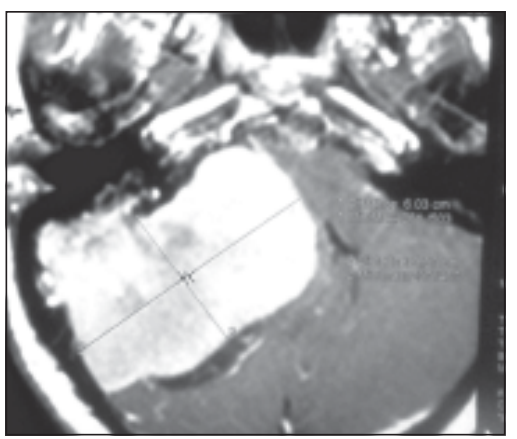

Fig.-3: Shows pre-op posterior petrosal meningioma.

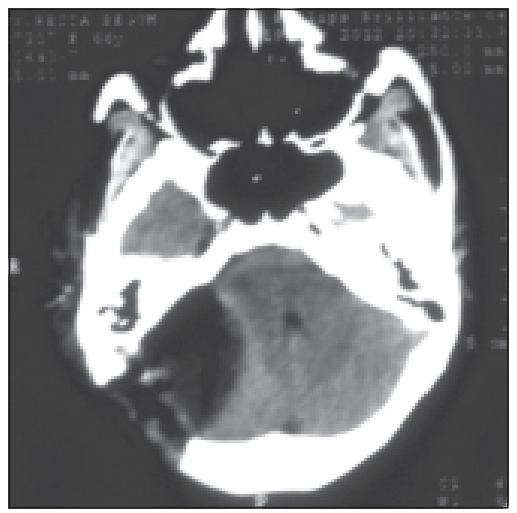

Fig.-4: Shows post-op picture of posterior petrosal meningioma.

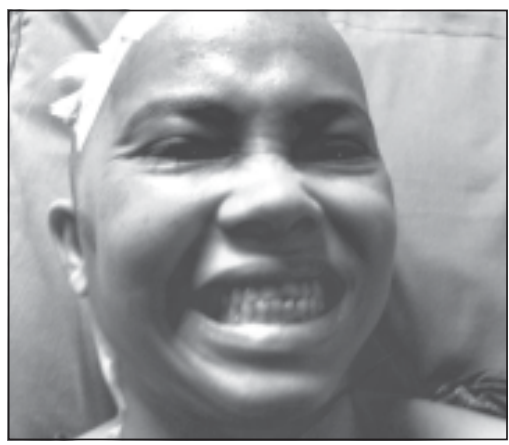

Fig.-5: Shows post-op picture of face showing no weakness. 
The highest concern is the risk of VAE and its sequelae. Although there are several published reports of the incidence of VAE in the sitting of adults, there are no large series that look at the incidence of VAE in children. The reported incidence as detected by transoesophageal doppler ultrasonography in adults ranges from 7 to $50 \%$. In another study its incidence is $35 \% \%^{9,10}$.

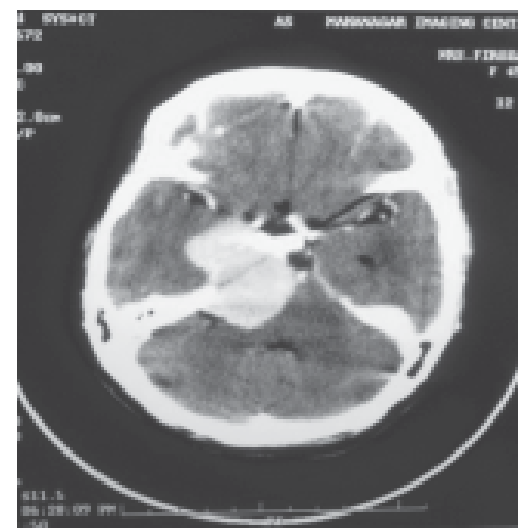

Fig.-6: Shows preoperative picture of petroclival meningioma.

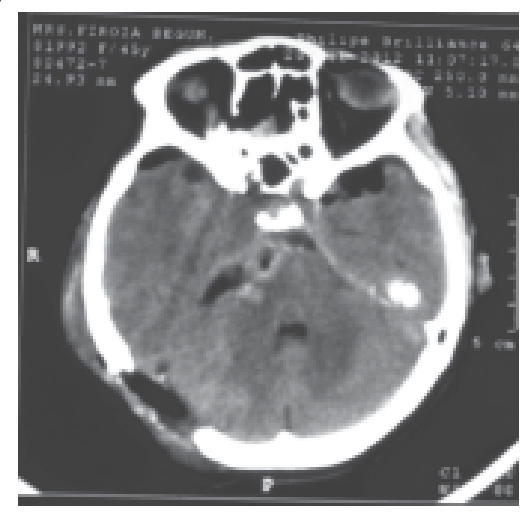

Fig.-7: Shows post operative picture of petroclival meningioma showing small residual tumour.

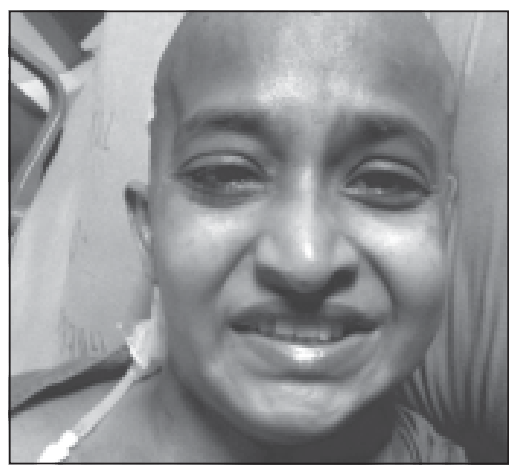

Fig.-8: Shows postoperative picture of having no facial weakness.
In our study venous air embolism occurred in 2 patients (6.6\%), which is still lower than other the previous reports. We believe that the care taken while positioning the patient and the meticulous prevention of bleeding from surgically opened venous vessels \& bony emissary vein were responsible for the lower air embolism rate ${ }^{10}$. For quick detection and management we have routinely used precordial Doppler(Figure -9) and cavafix(peripheral inserted central venous line (Figure-10).

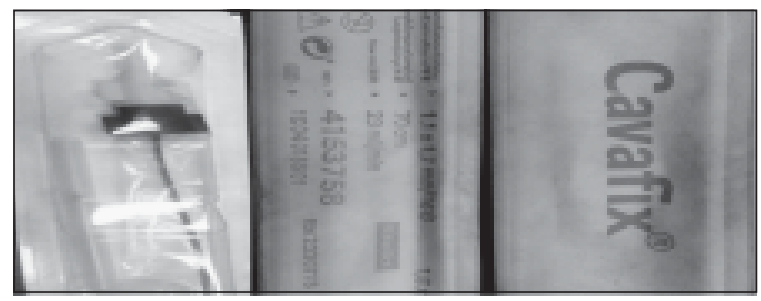

Fig.-9: Shows pheripheral inserted central venous catheter.

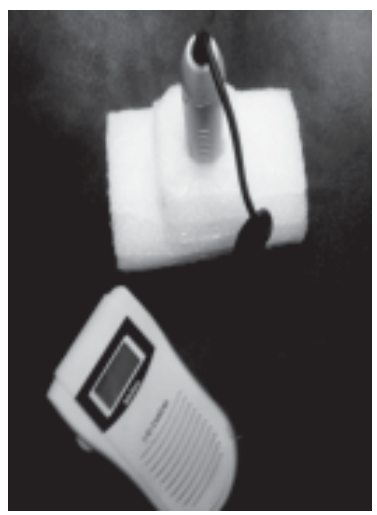

Fig.-10: Shows fetal doppler used as precordial doppler over the chest.

The effect of an air embolus depends both upon the rate and volume of air introduced into the circulation. The capacity of the lung to filter microbubbles of air from the venous circulation is exceeded when gas enters the circulatory system at a rate greater than $0.30 \mathrm{ml} / \mathrm{kg}$ per minute in a canine model; infusions at greater rates generally result in arterial emboli and tissue ischemia ${ }^{11}$.

Large, rapid boluses of air are tolerated less well than slow infusions of small amounts of air. It is estimated that 300 to $500 \mathrm{ml}$ of gas introduced at a rate of $100 \mathrm{ml} / \mathrm{sec}$ is a fatal dose for humans ${ }^{11}$. 
When air enters the veins, it travels to the right side of the heart, and then to the lungs. This can cause the vessels of the lung to constrict, raising the pressure in the right side of the heart. If the pressure rises high enough in a patient who is one of the $20-30 \%$ of the population with a patent foramen ovale, the gas bubble can then travel to the left side of the heart, and on to the brain or coronary arteries. Such bubbles are responsible for the most serious of gas embolic symptoms ${ }^{12}$.

Some studied have stated relative and absolute contraindications for the sitting position. Alongside age, hypertension and obstructive lung disease, diagnosed PFO is one of them. In a study by Kwapisz and colleagues the semi-sitting position chosen before operation was changed into supine position after diagnosing PFO to avoid complications ${ }^{12}$.

Contraindications to use the operative sitting position:

\section{Absolute:}

Patent ventriculo-atrial shunt.

Right atrial pressure in excess of left atrial pressure Patent foramen ovale.

Cerebral ischemia when upright and awake.

\section{Relative:}

Extremes of age.

Uncontrolled hypertension.

Chronic obstructive airway disease.

Certain pre-existing conditions may place patients at increased risk of venous air embolism (i.e. presence of a patent ventriculo-atrial shunt, demonstrable pressure gradient from left to right heart or presence of a patent foramen ovale). Patients who experienced cerebral ischemia in upright position as a result of cardiovascular and cerebrovascular disease are at increased risk of inadequate cerebral perfusion under anesthesia in the operative sitting position. Relative contraindications may include extremes of age, uncontrolled hypertension or chronic obstructive air way disease ${ }^{12}$.

\section{Conclusion:}

Sitting position can be safely done with good preoperative physiological, peroperative close monitoring of the patient regarding blood pressure, $\mathrm{ETCO}_{2}$ and oxygen saturation. However, postoperative complications like tumour bed haematoma, pneumocephalus, and cranial nerve palsy have to be bring in mind. Preoperative echocardiography investigation for detection of PFO further reduces the risk to the patient in that knowledge of the presence of PFO will heighten the surgeons degree of care and focus attention on alternative neurosurgical positions for surgery.

\section{References:}

1. Samii M, Matthies C. Management of 1000 vestibular schwannomas (acoustic neuromas): Surgical management and results with an emphasis on complications and how to avoid them. Neurosurgery 1997;40:11-21.

2. Yamakami I, Uchino Y, Kobayashi E, Yamaura A, Oka N. Removal of large acoustic neurinomas (vestibular schwannomas) by the retrosigmoid approach with no mortality and minimal morbidity. J Neurol Neurosurg Psychiatry 2004;75:453-8.

3. Martin JT. Neuroanesthetic adjuncts for surgery in the sitting position. I. Introduction and basic equipment. Anesth Analg. 1970;49(4):577-587.

4. Millar RA. Neurosurgical anaesthesia in the sitting position. A report of experience with 110 patients using controlled or spontaneous ventilation. Br J Anaesth. 1972;44(5):495-505.

5. Tindall GT, Craddock A, Greenfield JC., Jr. Effects of the sitting position on blood flow in the internal carotid artery of man during general anesthesia. J Neurosurg. 1967;26(4):383-9.

6. Michenfelder JD, Miller RH, Gronert GA. Evaluation of an ultrasonic device (Doppler) for the diagnosis of venous air embolism. Anesthesiology. 1972;36(2):164-7.

7. Maroon JC, Albin MS. Air embolism diagnosed by Doppler ultrasound. Anesth Analg. 1974;53(3):399-402.

8. Munson ES, Merrick HC. Effect of nitrous oxide on venous air embolism. Anesthesiology. 1966;27(6):783-7. 
9. Hitselberger WE, House WF: A warning regarding the sitting position for acoustic tumour surgery. Arch Otolaryngol 1980; 106:69.

10. BENGOCHEA FG, FERNANDEZ JC. The lateral sitting position for operations in the posterior fossa and in the cervical and upper thoracic regions of the spine. J Neurosurg. 1956;13(5):520-22.
11. Slbin MS, Babinski M, Maroon JC, Jannetta PJ. Anesthetic management of posterior fossa surgery in the sitting position. Acta Anaesthesiol Scand. 1976;20(2):117-28.

12. Kwapisz MM, Deinsberger W, Mueller M. Transesophageal echocardiography as a guide for patient positioning before neurosurgical procedures in semi-sitting position. J Neurosurg Anesthesiol 2004;4:277-81. 